\title{
Near-field imaging of perfectly conducting grating surfaces
}

\author{
Ting Cheng, ${ }^{1}$ Peijun $\mathrm{Li}^{2, * *}$ and Yuliang Wang ${ }^{2}$ \\ ${ }^{1}$ School of Mathematics and Statistics, Central China Normal University, Wuhan, Hubei 430079, China \\ ${ }^{2}$ Department of Mathematics, Purdue University, West Lafayette, Indiana 47907, USA \\ ${ }^{*}$ Corresponding author: lipeijun@math.purdue.edu
}

Received August 23, 2013; revised October 11, 2013; accepted October 13, 2013;

posted October 14, 2013 (Doc. ID 196341); published November 8, 2013

\begin{abstract}
A novel approach is presented to solving the inverse diffractive grating problem in near-field optical imaging, which is to reconstruct perfectly conducting grating surfaces with resolution beyond the diffraction limit. The grating surface is assumed to be a small and smooth deformation of a plane surface. An analytical solution of the direct grating problems is derived by using the method of transformed field expansion. Based on the analytic solution, an explicit reconstruction formula is deduced for the inverse grating problem. The method requires only a single incident field and is realized efficiently by using the fast Fourier transform. Numerical results show that the method is simple, stable, and effective in reconstructing grating surfaces with superresolved resolution. (c) 2013 Optical Society of America
\end{abstract}

OCIS codes: (050.1950) Diffraction gratings; (290.3200) Inverse scattering.

http://dx.doi.org/10.1364/JOSAA.30.002473

\section{INTRODUCTION}

We consider the diffraction when a time-harmonic electromagnetic plane wave is incident on some periodic structure, which is referred to as diffractive grating. Scattering theory in periodic structures has many applications in micro-optics, including the design and fabrication of optical elements such as corrective lenses, antireflective interfaces, beam splitters, and sensors. Depending on the direction and polarization of the incident plane wave, the governing model for the diffraction of time-harmonic electromagnetic waves can be simplified from the full three-dimensional Maxwell equations to two fundamental polarizations: the transverse electric (TE) polarization and the transverse magnetic (TM) polarization. In both polarizations, the scalar components of electromagnetic waves satisfy the two-dimensional Helmholtz equation. We refer to the monograph [1] for a good introduction to the problems of electromagnetic diffraction.

Recently, the scattering problems in periodic structures have received considerable attention in the applied mathematical community, and have been studied extensively on both mathematical and numerical aspects. We refer to Bao et al. [2] and the references therein for mathematical studies of the existence and uniqueness of the diffractive grating problems. Numerical methods can be found in [3-5] for either boundary integral equation approaches or variational approaches. A recent review can be found in Bao et al. [6] on mathematical modeling and computational methods.

The purpose of this paper is to develop an efficient numerical method for solving the inverse diffractive grating problem. Specifically, the inverse problem is to reconstruct the grating surface from a measured data field at a constant height above the grating structure. The mathematical questions on uniqueness and stability for the inverse problem have been studied by many researchers, e.g., Kirsch [7], Bao [8], Ammari [9], Hettlich and Kirsch [10], Bao and Friedman [11], Bao and Zhou [12], Bao et al. [13], and Bruckner et al. [14], for either the two-dimensional Helmholtz equation or the threedimensional Maxwell equations. Computationally, a number of numerical methods have been developed for the reconstruction of perfectly conducting grating surfaces in the TE polarization, e.g., García and Nieto-Vesperinas [15], Ito and Reitich [16], Arens and Kirsch [17], Hettlich [18], Bruckner and Elschner [19], And Elschner et al. [20]. Recently, Bao et al. [21,22] presented an efficient continuation method in order to capture fine structures of grating profiles with multiple frequency data with or without phase information. We refer to Dobson [23,24] and Elschner and Schmidt $[25,26]$ for related optimal design problems in diffractive optics, which are to design grating structures to obtain some specified diffraction patterns. Regardless of various reconstruction methods, the resolution is limited to half the wavelength, referred to as the Rayleigh criterion or the diffraction limit. It is a challenging task to achieve a stable reconstruction with subwavelength resolution due to the nonlinearity and ill-posedness of the problem.

By adopting a technique in near-field imaging [27], we develop an efficient computational method for solving the inverse grating surface problem with a resolution beyond the diffraction limit. For clarity, we shall concentrate on the TE polarization since the discussion is analogous for the TM polarization. The more complicated biperiodic diffractive grating problem will be considered as future work. The grating surface is assumed to be a small and smooth deformation of a plane surface. The method begins with the transformed field expansion to analytically derive the solution for the direct problem; by neglecting higher-order terms in power series expansion, the nonlinear inverse problem is 
linearized and an explicit reconstruction formula is deduced. It requires only a single frequency data field from one incident plane wave, and is realized by the fast Fourier transform. Numerical results show that the method is simple, stable, and effective in reconstructing grating surfaces with subwavelength resolution. For transformed field expansion or the related boundary perturbation method, we refer to Nicholls and Reitich [28,29], Bruno and Reitich [30], Malcolm and Nicholls [31], and $\mathrm{Li}$ and Shen [32] for solving the direct diffraction grating problem and the direct unbounded rough surface scattering problem, respectively. A boundary perturbation method may be found in Malcolm and Nicholls [33] for solving an inverse scattering problem with a periodic surface. We refer to Bao and Lin [34] and Bao and $\mathrm{Li}$ [35] for related work on near-field imaging of locally perturbed and infinite rough surfaces, respectively.

The outline of this paper is as follows. The mathematical model problem of the diffractive grating is introduced in Section $\underline{2}$. Sections $\underline{3}$ and $\underline{4}$ present the transformed field expansion and Fourier series expansion to deduce an analytic solution for the direct problem. Section $\underline{5}$ is devoted to the derivation of an explicit reconstruction formula for the inverse problem. Numerical examples are reported in Section $\underline{6}$. The paper is concluded with some remarks and direction for future work in Section $\underline{7}$.

\section{MODEL PROBLEM}

Let us first specify the problem geometry. As seen in Fig. 1, the problem may be restricted to a single period of $\Lambda$ in $x$ due to the periodicity of the grating surface. Let the grating surface in one period be described by the curve

$$
S=\left\{(x, y) \in \mathbb{R}^{2}: y=f(x), 0<x<\Lambda\right\},
$$

where $f$ is a periodic function with period $\Lambda$ and is assumed to take the form

$$
f(x)=\varepsilon g(x), \quad g \in C^{2}(\mathbb{R}) .
$$

Here $\varepsilon$ is a sufficiently small positive constant. Let the space above $S$ be filled with a homogeneous medium with a positive constant wavenumber $\kappa$.

Denote by

$$
\Omega=\left\{(x, y) \in \mathbb{R}^{2}: f(x)<y<h, 0<x<\Lambda\right\}
$$

the domain bounded below by $S$ and bounded above by

$$
\Gamma=\left\{(x, y) \in \mathbb{R}^{2}: y=h, 0<x<\Lambda\right\},
$$

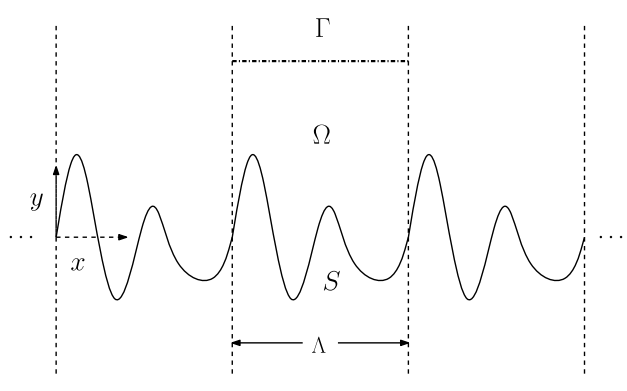

Fig. 1. Problem geometry. where $h>0$ is a constant satisfying $h>\max _{0<x<\Lambda} f(x)$ and is denoted as the measurement distance.

Let an incoming plane wave $u^{\text {inc }}=e^{\mathrm{i}(\alpha x-\beta y)}$ be incident on the grating surface from above, where $\alpha=\kappa \sin \theta$, $\beta=\kappa \cos \theta$, and $\theta \in(-\pi / 2, \pi / 2)$ is the incident angle. For normal incidence, i.e., $\theta=0$, we have $\alpha=0$ and $\beta=\kappa$. The incident field reduces to $u^{\mathrm{inc}}=e^{-\mathrm{i} \kappa y}$. For simplicity, we shall focus on the normal incidence from now on since our method requires only a single incident wave.

The diffraction of a time-harmonic electromagnetic wave in the TE polarization can be modeled by the two-dimensional Helmholtz equation:

$$
\Delta u+\kappa^{2} u=0 \quad \text { in } \Omega
$$

For a perfectly conducting grating, the total field $u$ vanishes on the grating surface:

$$
u=0 \quad \text { on } S \text {. }
$$

Motivated by uniqueness, we shall seek the periodic solution of $u$. Moreover, the diffracted field $u^{\mathrm{d}}$ is required to be a bounded outgoing wave in $\Omega$. It follows from Rayleigh's expansion that $u$ can be written as a sum of plane waves:

$$
u=u^{\mathrm{inc}}+u^{\mathrm{d}}=e^{-\mathrm{i} \kappa y}+\sum_{n \in \mathbb{Z}} A_{n} e^{\mathrm{i} \alpha_{n} x+\mathrm{i} \beta_{n} y},
$$

for $y>\max _{0<x<\Lambda} f(x)$, where $A_{n}$ is a complex number,

$$
\alpha_{n}=n\left(\frac{2 \pi}{\Lambda}\right), \quad \beta_{n}=\left\{\begin{array}{cc}
\left(\kappa^{2}-\alpha_{n}^{2}\right)^{1 / 2} & \text { for } \kappa>\left|\alpha_{n}\right|, \\
\mathrm{i}\left(\alpha_{n}^{2}-\kappa^{2}\right)^{1 / 2} & \text { for } \kappa<\left|\alpha_{n}\right|
\end{array}\right.
$$

For any given periodic function $q(x)$ with period $\Lambda$, it has a Fourier series expansion

$$
q(x)=\sum_{n \in \mathbb{Z}} q_{n} e^{\mathrm{i} \alpha_{n} x}, \quad q_{n}=\frac{1}{\Lambda} \int_{0}^{\Lambda} q(x) e^{-\mathrm{i} \alpha_{n} x} \mathrm{~d} x .
$$

We define a boundary operator

$$
(T q)(x)=\sum_{n \in \mathbb{Z}} \mathrm{i} \beta_{n} q_{n} e^{\mathrm{i} \alpha_{n} x}
$$

Using Eq. (3), we may derive a transparent boundary condition for the total field:

$$
\partial_{y} u=T u+\rho \quad \text { on } \Gamma
$$

where $\rho=-2 \mathrm{i} \kappa e^{-\mathrm{i} \kappa h}$.

This paper aims to study the inverse diffractive grating problem, which is to reconstruct the grating surface function $f$ from the measurement of the total field $u$ on $\Gamma$, given the incident field $u^{\text {inc }}$. In particular, we are interested in the inverse problem in the near-field regime where the measurement distance $h$ is much smaller than the wavelength $\lambda=2 \pi / \kappa$ of the incident wave. 


\section{TRANSFORMED FIELD EXPANSION}

The transformed field expansion method begins with the changes of variables:

$$
\tilde{x}=x, \quad \tilde{y}=h\left(\frac{y-f}{h-f}\right),
$$

which maps the domain $\Omega$ to the rectangle

$$
D=\left\{(\tilde{x}, \tilde{y}) \in \mathbb{R}^{2}: 0<\tilde{y}<h, 0<x<\Lambda\right\}
$$

Introduce a new function $w(\tilde{x}, \tilde{y})=u(x, y)$ under the transformation. It can be verified, after tedious but straightforward calculations from Eq. (1), that $w$, upon dropping the tilde, satisfies the equation in $D$ :

$$
c_{1} \frac{\partial^{2} w}{\partial x^{2}}+c_{2} \frac{\partial^{2} w}{\partial y^{2}}+c_{3} \frac{\partial^{2} w}{\partial x \partial y}+c_{4} \frac{\partial w}{\partial y}+c_{1} \kappa^{2} w=0
$$

where

$$
\begin{aligned}
& c_{1}=(h-f)^{2}, \\
& c_{2}=\left[f^{\prime}(h-y)\right]^{2}+h^{2}, \\
& c_{3}=-2 f^{\prime}(h-y)(h-f), \\
& c_{4}=-(h-y)\left[f^{\prime \prime}(h-f)+2\left(f^{\prime}\right)^{2}\right] .
\end{aligned}
$$

The perfectly conducting condition (2) becomes

$$
w(x, y)=0 \quad \text { on } y=0 \text {. }
$$

The transparent boundary condition (4) reduces to

$$
\partial_{y} w=\left(1-\frac{f}{h}\right)(T w+\rho) \quad \text { on } y=h .
$$

Due to the small perturbation assumption, we may consider a formal expansion of $w$ in a power series of $\varepsilon$ :

$$
w(x, y ; \varepsilon)=\sum_{k=0}^{\infty} w_{k}(x, y) \varepsilon^{k}
$$

Substituting $f=\varepsilon g$ into $c_{j}$ and inserting the power series expansion (8) into Eqs. (5)-(7), we may derive the recursion equation for $w_{k}$ :

$$
\frac{\partial^{2} w_{k}}{\partial x^{2}}+\frac{\partial^{2} w_{k}}{\partial y^{2}}+\kappa^{2} w_{k}=v_{k} \quad \text { in } D,
$$

where

$$
\begin{aligned}
v_{k}= & \frac{2 g}{h} \frac{\partial^{2} w_{k-1}}{\partial x^{2}}+\frac{2 g^{\prime}(h-y)}{h} \frac{\partial^{2} w_{k-1}}{\partial x \partial y}+\frac{g^{\prime \prime}(h-y)}{h} \frac{\partial w_{k-1}}{\partial y} \\
& +\frac{2 \kappa^{2} g}{h} w_{k-1}-\frac{g^{2}}{h^{2}} \frac{\partial^{2} w_{k-2}}{\partial x^{2}}-\frac{\left(g^{\prime}\right)^{2}(h-y)^{2}}{h^{2}} \frac{\partial^{2} w_{k-2}}{\partial y^{2}} \\
& -\frac{2 g g^{\prime}(h-y)}{h^{2}} \frac{\partial^{2} w_{k-2}}{\partial x \partial y}+\frac{\left[2\left(g^{\prime}\right)^{2}-g g^{\prime \prime}\right](h-y)}{h^{2}} \frac{\partial w_{k-2}}{\partial y} \\
& -\frac{\kappa^{2} g^{2}}{h^{2}} w_{k-2},
\end{aligned}
$$

together with boundary conditions

$$
w_{k}(x, y)=0 \quad \text { on } y=0
$$

and

$$
\partial_{y} w_{k}-T w_{k}=\rho_{k} \quad \text { on } y=h,
$$

where

$$
\rho_{0}=\rho,
$$

$$
\begin{aligned}
& \rho_{1}=-\left(\frac{g}{h}\right)\left(T w_{0}+\rho\right), \\
& \rho_{k}=-\left(\frac{g}{h}\right) T w_{k-1} .
\end{aligned}
$$

In Eqs. (9)-(15), the recursion equation of $w_{k}$ involves $v_{k}$ and $\rho_{k}$. They only depend on the previous two terms $w_{k-1}$ and $w_{k-2}$, where $w_{-1}=w_{-2}=0$. Thus, the scattering problem (9)-(15) can indeed be solved in a recursive manner starting from $k=0$.

\section{FOURIER SERIES EXPANSION}

Since $w_{k}, v_{k}, \rho_{k}$ are periodic functions in $x$ with period $\Lambda$, they have the following Fourier series expansions:

$$
\begin{aligned}
w_{k}(x, y) & =\sum_{n \in \mathbb{Z}} w_{k}^{(n)}(y) e^{\mathrm{i} \alpha_{n} x}, \\
v_{k}(x, y) & =\sum_{n \in \mathbb{Z}} v_{k}^{(n)}(y) e^{\mathrm{i} \alpha_{n} x}, \\
\rho_{k}(x) & =\sum_{n \in \mathbb{Z}} \rho_{k}^{(n)} e^{\mathrm{i} \alpha_{n} x} .
\end{aligned}
$$

Substituting these expansions into Eqs. (9)-(15), we obtain a two-point boundary value problem for $w_{k}^{(n)}$ :

$$
\begin{gathered}
\frac{\mathrm{d}^{2} w_{k}^{(n)}}{\mathrm{d} y^{2}}+\beta_{n}^{2} w_{k}^{(n)}=v_{k}^{(n)}, \quad 0<y<h, \\
w_{k}^{(n)}=0 \quad \text { at } y=0, \\
\frac{\mathrm{d} w_{k}^{(n)}}{\mathrm{d} y}-\mathrm{i} \beta_{n} w_{k}^{(n)}=\rho_{k}^{(n)} \quad \text { at } y=h .
\end{gathered}
$$

Using Theorem B.1, we may obtain an explicit solution of Eqs. (16)-(18).

Theorem 4.1. The two-point boundary value problem (16)-(18) has a unique solution, which is given by

$$
w_{k}^{(n)}(y)=K_{1}^{(n)}(y) \rho_{k}^{(n)}-\int_{0}^{h} K_{2}^{(n)}(y, z) v_{k}^{(n)}(z) \mathrm{d} z,
$$

where

$$
K_{1}^{(n)}(y)=\frac{e^{\mathrm{i} \beta_{n} h}}{2 \mathrm{i} \beta_{n}}\left(e^{\mathrm{i} \beta_{n} y}-e^{-\mathrm{i} \beta_{n} y}\right)
$$


and

$$
K_{2}^{(n)}(y, z)= \begin{cases}\frac{e^{\mathrm{i} \beta_{n} y}}{2 \mathrm{i} \beta_{n}}\left(e^{\mathrm{i} \beta_{n} z}-e^{-\mathrm{i} \beta_{n} z}\right), & z<y, \\ \frac{e^{\mathrm{i} \beta} z}{2 \mathrm{i} \beta_{n}}\left(e^{\mathrm{i} \beta_{n} y}-e^{-\mathrm{i} \beta_{n} y}\right), & z>y .\end{cases}
$$

It follows from Theorem 4.1 that the power series (8) along with the solution representation (19) gives an analytic solution of the direct grating problem (5) $-(7)$.

\section{RECONSTRUCTION FORMULA}

Assume that the noisy data take the form

$$
u_{\delta}(x, h)=u(x, h)+O(\delta),
$$

where $u(x, h)$ is the noise-free data and $\delta$ represents the noise level.

Using Eq. (ㅁ), we have

$$
w(x, y)=w_{0}(x, y)+\varepsilon w_{1}(x, y)+O\left(\varepsilon^{2}\right) .
$$

Evaluating Eq. (23) at $y=h$, and noting $w(x, h)=u(x, h)$ and $w_{\delta}(x, h)=u_{\delta}(x, \bar{h})$, we have

$$
w_{\delta}(x, h)=w_{0}(x, h)+\varepsilon w_{1}(x, h)+O\left(\varepsilon^{2}\right)+O(\delta) .
$$

Rearranging Eq. (24) yields

$$
\varepsilon w_{1}(x, h)=\left(w_{\delta}(x, h)-w_{0}(x, h)\right)+O\left(\varepsilon^{2}\right)+O(\delta),
$$

which is the basis of our inversion formula. Here the two parameters $\varepsilon$ and $\delta$ indicate the ill-posed nature of the inverse problem; the larger the two parameters $\varepsilon$ and $\delta$ are, the severer the ill-posedness of the inverse problem is. Neglecting the terms of $\varepsilon^{2}$ and $\delta$ in Eq. (25) yields

$$
\varepsilon w_{1}(x, h)=w_{\delta}(x, h)-w_{0}(x, h),
$$

which actually linearizes the inverse problem and may lead to an explicit inversion formula for the linearized inverse problem.

Based on Eq. (26), we next shall derive the analytical solution for the leading term $w_{0}$, deduce an equation relating $w_{1}$ and the scattering surface function $f$, and obtain an explicit inversion formula.

Recalling Eqs. (느) and (13), we have

$$
v_{0}=0, \quad \rho_{0}=-2 \mathrm{i} \kappa e^{-\mathrm{i} \kappa h},
$$

whose Fourier coefficients are

$$
v_{0}^{(n)}=0, \quad \rho_{0}^{(n)}=\left\{\begin{array}{cc}
-2 \mathrm{i} \kappa e^{-\mathrm{i} \kappa h}, & n=0, \\
0, & n \neq 0 .
\end{array}\right.
$$

Using Eq. (19) and noting $\beta_{0}=\beta$, we get

$$
\begin{aligned}
w_{0}^{(n)}(y) & =K_{1}^{(n)}(y) \rho_{0}^{(n)}=\frac{e^{\mathrm{i} \kappa h}}{2 \mathrm{i} \kappa}\left(e^{\mathrm{i} \kappa y}-e^{-\mathrm{i} \kappa y}\right) \rho_{0}^{(n)} \\
& =\left\{\begin{array}{cl}
e^{-\mathrm{i} \kappa y}-e^{\mathrm{i} \kappa y}, & n=0, \\
0, & n \neq 0,
\end{array}\right.
\end{aligned}
$$

which yields

$$
w_{0}(x, y)=\sum_{n \in \mathbb{Z}} w_{0}^{(n)}(y) e^{\mathrm{i} \alpha_{n} x}=e^{-\mathrm{i} \kappa y}-e^{\mathrm{i} \kappa y} .
$$

Recalling Eqs. (14) and (12), we obtain

$$
\rho_{1}(x)=-\left(\frac{g}{h}\right) \partial_{y} w_{0}(x, h)=2 \mathrm{i} \kappa h^{-1} \cos (\kappa h) g(x),
$$

whose Fourier coefficients are given by

$$
\rho_{1}^{(n)}=2 \mathrm{i} \kappa h^{-1} \cos (\kappa h) g_{n},
$$

where $g_{n}$ is the Fourier coefficient of $g(x)$.

It follows from Eq. (10) that we have

$$
\begin{aligned}
v_{1}(x, y)= & \frac{2 g}{h} \frac{\partial^{2} w_{0}}{\partial x^{2}}+\frac{2 g^{\prime}(h-y)}{h} \frac{\partial^{2} w_{0}}{\partial x \partial y} \\
& +\frac{g^{\prime \prime}(h-y)}{h} \frac{\partial w_{0}}{\partial y}+\frac{2 \kappa^{2} g}{h} w_{0}
\end{aligned}
$$

Substituting $w_{0}$ into the above equation yields

$$
v_{1}=-2 \mathrm{i} \kappa h^{-1}(h-y) \cos (\kappa y) g^{\prime \prime}(x)-4 \mathrm{i}^{2} h^{-1} \sin (\kappa y) g(x),
$$

whose Fourier coefficient is

$$
v_{1}^{(n)}=\left[-4 \mathrm{i} \kappa^{2} h^{-1} \sin (\kappa y)+2 \mathrm{i} \kappa h^{-1}(h-y) \alpha_{n}^{2} \cos (\kappa y)\right] g_{n} .
$$

Following Eq. (19), we get

$$
w_{1}^{(n)}(y)=K_{1}^{(n)}(y) \rho_{1}^{(n)}-\int_{0}^{h} K_{2}^{(n)}(y, z) v_{1}^{(n)}(z) \mathrm{d} z .
$$

Since the data are measured at $\Gamma$, we evaluate Eq. (28) at $y=h$ and obtain

$$
w_{1}^{(n)}(h)=K_{1}^{(n)}(h) \rho_{1}^{(n)}-\int_{0}^{h} K_{2}^{(n)}(h, z) v_{1}^{(n)}(z) \mathrm{d} z
$$

Using the expressions of $\rho_{1}^{(n)}$ and $v_{1}^{(n)}$, and substituting Eqs. (19)-(21) into Eq. (29), yields

$$
\begin{aligned}
w_{1}^{(n)}(h)= & \frac{\kappa e^{\mathrm{i} \beta_{n} h}}{\beta_{n} h}\left(e^{\mathrm{i} \beta_{n} h}-e^{-\mathrm{i} \beta_{n} h}\right) \cos (\kappa h) g_{n} \\
& +\frac{\kappa e^{\mathrm{i} \beta_{n} h}}{\beta_{n} h} \int_{0}^{h}\left(e^{\mathrm{i} \beta_{n} z}-e^{-\mathrm{i} \beta_{n} z}\right) \\
& \times\left[2 \kappa \sin (\kappa z)-\alpha_{n}^{2}(h-z) \cos (\kappa z)\right] g_{n} \mathrm{~d} z .
\end{aligned}
$$

Using integration by parts and the identity $\alpha_{n}^{2}+\beta_{n}^{2}=\kappa^{2}$, we have

$$
\begin{aligned}
\int_{0}^{h}\left(e^{\mathrm{i} \beta_{n} z}-e^{-\mathrm{i} \beta_{n} z}\right) \sin (\kappa z) \mathrm{d} z= & \frac{\mathrm{i} \beta_{n}}{\alpha_{n}^{2}}\left(e^{\mathrm{i} \beta_{n} h}+e^{-\mathrm{i} \beta_{n} h}\right) \sin (\kappa h) \\
& -\frac{\kappa}{\alpha_{n}^{2}}\left(e^{\mathrm{i} \beta_{n} h}-e^{-\mathrm{i} \beta_{n} h}\right) \cos (\kappa h)
\end{aligned}
$$

and 


$$
\begin{aligned}
\int_{0}^{h}\left(e^{\mathrm{i} \beta_{n} z}-e^{-\mathrm{i} \beta_{n} z}\right)(h-z) \cos (\kappa z) \mathrm{d} z \\
=-\frac{\left(\kappa^{2}+\beta_{n}^{2}\right)}{\alpha_{n}^{4}}\left(e^{\mathrm{i} \beta_{n} h}-e^{-\mathrm{i} \beta_{n} h}\right) \cos (\kappa h)+\frac{2 \mathrm{i} \kappa \beta_{n}}{\alpha_{n}^{4}} \\
\quad \times\left(e^{\mathrm{i} \beta_{n} h}+e^{-\mathrm{i} \beta_{n} h}\right) \sin (\kappa h)-\frac{2 \mathrm{i} \beta_{n} h}{\alpha_{n}^{2}} .
\end{aligned}
$$

Substituting the above two identities into Eq. (30), we obtain

$$
w_{1}^{(n)}(h)=2 \mathrm{i} \kappa e^{\mathrm{i} \beta_{n} h} g_{n}
$$

Note $f=\varepsilon g$ and thus $f_{n}=\varepsilon g_{n}$, where $f_{n}$ is the Fourier coefficient of $f$. Combining Eqs. (26) and (31), we deduce

$$
f_{n}=-\frac{\mathrm{i}}{2 \kappa}\left(w_{\delta}^{(n)}(h)-w_{0}^{(n)}(h)\right) e^{-\mathrm{i} \beta_{n} h},
$$

where $w_{\delta}^{(n)}(h)$ is the Fourier coefficient of the noise data $w_{\delta}(x, h)$ and

$$
w_{0}^{(n)}(h)=\left\{\begin{array}{cc}
e^{-\mathrm{i} \kappa h}-e^{\mathrm{i} \kappa h}, & n=0 \\
0, & n \neq 0 .
\end{array}\right.
$$

It follows from the definition of $\beta_{n}$ and Eq. (32) that it is wellposed to reconstruct those Fourier coefficients $f_{n}$ with $\left|\alpha_{n}\right|<\kappa$, since the small variations of the measured data will not be amplified and lead to large errors in the reconstruction, but the resolution of the reconstructed function $f$ is restricted by the given wavenumber $\kappa$. In contrast, it is severely ill-posed to reconstruct those Fourier coefficients $f_{n}$ with $\left|\alpha_{n}\right|>\kappa$, since the small variations in the data will be exponentially enlarged and lead to huge errors in the reconstruction, but they contribute to the super resolution of the reconstructed function $f$.

To obtain a stable and super-resolved reconstruction, we may adopt a regularization to suppress the exponential growth of the reconstruction errors. Besides, we may use as small an $h$ as possible, i.e., measure the data at the distance that is as close as possible to the grating surface, which is exactly the idea of near-field optics.

Following [34], we consider the spectral cut-off regularization. Define the signal-to-noise ratio (SNR) by

$$
\mathrm{SNR}=\min \left\{\varepsilon^{-2}, \delta^{-1}\right\} .
$$

For fixed $h$, the cut-off frequency $\omega$ is chosen in such a way that

$$
e^{\left(\omega^{2}-\kappa^{2}\right)^{1 / 2} h}=\mathrm{SNR},
$$

which implies that the spatial frequency will be cut-off for those below the noise level. More explicitly, we have

$$
\frac{\omega}{\kappa}=\left[1+\left(\frac{\log \mathrm{SNR}}{\kappa h}\right)^{2}\right]^{1 / 2},
$$

which indicates $\omega>\kappa$ as long as SNR $>0$ and super resolution may be achieved.
Taking into account the frequency cut-off, we may have a regularized inversion formulation for Eq. (32):

$$
f_{n}=-\frac{\mathrm{i}}{2 \kappa}\left(w_{\delta}^{(n)}(h)-w_{0}^{(n)}(h)\right) e^{-\mathrm{i} \beta_{n} h} \chi_{[-\omega, \omega]},
$$

where the characteristic function

$$
\chi_{[-\omega, \omega]}\left(\alpha_{n}\right)= \begin{cases}1 & \text { for }\left|\alpha_{n}\right| \leq \omega \\ 0 & \text { for }\left|\alpha_{n}\right|>\omega\end{cases}
$$

Once $f_{n}$ are computed, the grating surface function can be approximated by

$$
f(x) \approx \sum_{\left|\alpha_{n}\right| \leq \omega} f_{n} e^{\mathrm{i} \alpha_{n} x}
$$

Hence, only two fast Fourier transforms are needed to reconstruct the grating surface function: one is done for the data to obtain $w_{\delta}^{(n)}(h)$, and another is done to obtain the approximated function $f$.

\section{NUMERICAL EXPERIMENTS}

Here we present two numerical examples to illustrate the effectiveness of our method. As a constant height configuration, the near-field data $u(x, h)$ are simulated by solving the direct grating problem using the finite element method with a perfectly matched layer. The wavenumber is taken as $\kappa=2 \pi$, which corresponds to the wavelength $\lambda=1$. The grating period is $\Lambda=1$, i.e., $\Lambda=\lambda$. In all the figures, the plots are rescaled with respect to the wavelength $\lambda$. Due to the unstructured triangular meshes, the wave field data $u(x, h)$ are not equally spaced with respect to $x$. We construct a curve $u(x, h)$ by using the natural cubic spline interpolation formula based on the computed discrete data $u(x, h)$. The curve $u(x, h)$ is evaluated at equally spaced points $x_{j}, j=0,1, \ldots, 512$, in the interval [0,1], and is used as our synthetic scattering data. For the stability test, some relative random noise is added to the data; i.e., the nearfield measurement is updated with

$$
u_{\delta}(x, h)=u(x, h)(1+\delta \text { rand }),
$$

where rand represents a normally distributed random number in $[-1,1]$.

Example 1. The exact grating surface is given by $f(x)=\varepsilon g(x)$, where

$$
g(x)=0.5 \sin (2 \pi x)+0.5 \sin (6 \pi x) .
$$

This is a simple example since the grating profile function $g$ only contains a couple of low Fourier modes. We examine the dependence of reconstruction results on the parameters $h$ and $\varepsilon$. Figure 2 plots the reconstructed surfaces (dashed lines) against the exact surfaces (solid lines). First we fix $\varepsilon=0.01, \delta=0.01$ and consider the reconstructions using two different measurement distances $h$. Figures $\underline{2(a)}$ and 2(b) plot the reconstructed surfaces by using $h=\overline{0.1 \lambda}$ and $h=0.2 \lambda$, respectively. It is clear that smaller $h$ in Fig. $\underline{2(a)}$ gives better reconstruction than larger $h$ in Fig. 2(b) does. In particular, the fine features of the grating surface are 

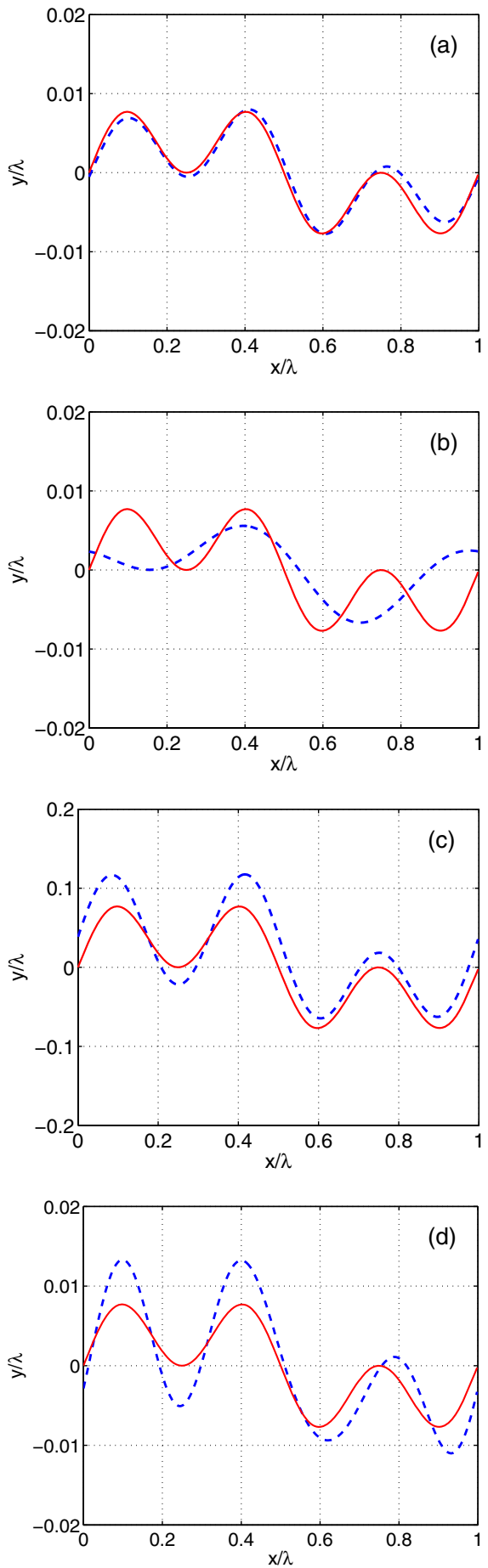

Fig. 2. Example 1: finite Fourier grating; the exact surface (solid line) is plotted against the reconstructed surface (dashed line) using different $h, \varepsilon, \delta$. (a) $h=0.1 \lambda, \varepsilon=0.01, \delta=0.01$; (b) $h=0.2 \lambda, \varepsilon=0.01$, $\delta=0.01$; (c) $h=0.1 \lambda, \varepsilon=0.1, \delta=0.01$; and (d) $h=0.1 \lambda, \varepsilon=0.01$, $\delta=0.05$.

completely recovered and the subwavelength resolution is achieved when using $h=0.1 \lambda$ in Fig. 2(a). This is attributed to the fact that larger cut-off frequency $\omega$ may be used in the reconstruction when the measurement distance $h$ is smaller. Next we fix $h=0.1 \lambda, \delta=0.01$ and consider reconstructions for two different $\varepsilon$. Figures 2(a) and 2(c) show the reconstruction surfaces by using $\varepsilon=\overline{0.01}$ and $\varepsilon=0.1$, respectively. Evidently, smaller $\varepsilon$ in Fig. 2(a) yields a better
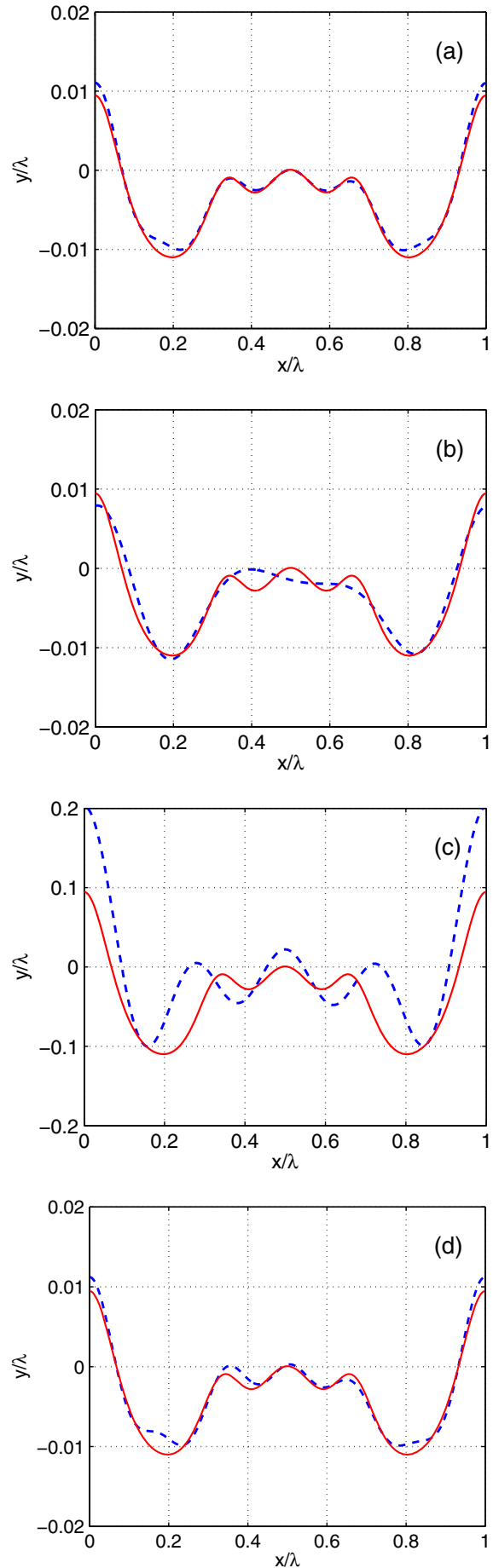

Fig. 3. Example 2: infinite Fourier grating; the exact surface (solid line) is plotted against the reconstructed surface (dashed line) using different $h, \varepsilon, \delta$. (a) $h=0.02 \lambda, \varepsilon=0.01, \delta=0.01$; (b) $h=0.1 \lambda$, $\varepsilon=0.01, \delta=0.01$; (c) $h=0.11 \lambda, \varepsilon=0.1, \delta=0.01$; and (d) $h=0.02 \lambda$, $\varepsilon=0.01, \delta=0.05$.

result than larger $\varepsilon$ in Fig. 2(c) does. The reason is that smaller $\varepsilon$ gives more accurate approxiation of the linearized model to the original nonlinear model problem. Finally we consider a higher level of noise $\delta$. Figure 1(d) plots the reconstructed grating surface by using $h=0.1 \overline{\lambda, \varepsilon}=0.01, \delta=0.05$. Comparing Figs. 1 (a) and $\underline{1(d)}$, we can still stably reconstruct the shape of the exact surface with $5 \%$ noise, though the result is not as good as that with $1 \%$ noise. 
Example 2. The exact grating surface is given by $f(x)=\varepsilon g(x)$, where

$$
g(x)=0.5 e^{\cos (4 \pi x)}+0.4 e^{\cos (6 \pi x)}-1.5 .
$$

This is a harder example as the grating profile function $g$ has infinite Fourier modes. It is expected that higher cut-off frequency $\omega$ is desirable and thus even smaller $h$ is necessary in order to capture all the surface features. Similarly, we investigate the effects of $h$ and $\varepsilon$ on the reconstructions. For fixed $\varepsilon=0.01$, Figs. 3(a) and 3(b) show the reconstructed surfaces

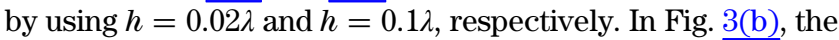
reconstruction is unable to capture the oscillatory middle part of the exact grating surface by using $h=0.1 \lambda$, which is enough for the grating surface in Example 1. In contrast, all the detailed features are reconstructed in Fig. 3(a) when using $h=0.02 \lambda$, because larger $\omega$ is indeed allowed to be taken. Next we compare the results by using different $\varepsilon$. In Fig. 3(a), the measurement distance $h=0.02 \lambda$, and the maximum value of the grating surface is approximately $0.01 \lambda$. So the measurement is taken at the place that is roughly $0.01 \lambda$ away from the grating structure. In Fig. 3(c), the deformation parameter $\varepsilon=0.1$, and the maximum value of the grating surface is about $0.1 \lambda$. Thus the data are measured at $h=0.11 \lambda$ in order to have the same measurement distance to the grating structure as that in Fig. 3(a). Again, it can be seen from Figs. 3(a) and 3(c) that smaller $\varepsilon$ yields better reconstruction than larger $\varepsilon \overline{\text { due }}$ to the approximation error in the linearization procedure. Figure $1(\mathrm{~d})$ plots the reconstructed result by using a higher level of noise $\delta=0.05$ and shows that the method is stable and can yield good results even for data with a higher level of noise.

\section{CONCLUSION}

We have presented a simple, stable, and effective computational method for solving the inverse diffractive grating surface problem and achieved subwavelength resolution. Using the transformed field expansion, we deduced an analytic solution for the direct problem. By dropping higher-order terms in power series, we linearized the nonlinear inverse problem and obtained an explicit reconstruction formula, which was implemented by using the fast Fourier transform. We considered two examples - one of which has finite Fourier modes, and the other has infinite Fourier modes-and investigated how the parameters influence the reconstructions. The results show that super resolution may be achieved by using a small measurement distance. The method works very well for the model problems arising from the near-field optical imaging where the structures are in the size of the subwavelength region. As for future work, we are to extend the proposed method to biperiodic structures, where the full threedimensional Maxwell equations should be considered, and will report the results elsewhere.

\section{APPENDIX A: INTEGRATION SOLUTION METHOD}

For self-containedness, the integrated solution method is briefly introduced to solve a two-point boundary value problem. We refer to Zhang [36] for details of the integrated solutions of the ordinary differential equation system and two-point boundary value problems.
Consider the two-point boundary value problem

$$
\mathbf{u}^{\prime}(y)+M(y) \mathbf{u}(y)=\mathbf{f}(y)
$$

$$
\left.A_{0} \mathbf{u}(y)\right|_{y=0}=\mathbf{r}_{0},
$$

$$
\left.B_{1} \mathbf{u}(y)\right|_{y=h}=\mathbf{s}_{1},
$$

where $\mathbf{f}(y) \in \mathbb{C}^{m}$ are $m$-dimensional vector fields, $\mathbf{r}_{0} \in \mathbb{C}^{m_{1}}$ and $\mathbf{s}_{1} \in \mathbb{C}^{m_{2}}$ are given $m_{1}$ - and $m_{2}$-dimensional vector fields, respectively, $M(y) \in \mathbb{C}^{m \times m}$ is an $m \times m$ matrix, and $A_{0} \in$ $\mathbb{C}^{m_{1} \times m}$ and $B_{1} \in \mathbb{C}^{m_{2} \times m}$ are full rank matrices with $m_{1}+m_{2}=m$, i.e., rank $A_{0}=m_{1}$ and $\operatorname{rank} B_{1}=m_{2}$.

Let $\Phi(y)$ be the fundamental matrix of the system:

$$
\begin{gathered}
\Phi^{\prime}(y)+M(y) \Phi(y)=\mathbf{0}, \\
\Phi(0)=I_{m},
\end{gathered}
$$

where $I_{m}$ is the $m \times m$ identity matrix.

Theorem A.1. The two-point boundary value problem (A.1)-(A.3) has a unique solution if and only if

$$
\operatorname{det}\left[\begin{array}{c}
A_{0} \\
B_{1} \Phi(h)
\end{array}\right] \neq 0 .
$$

Let the pair of functions $\{A(y), \mathbf{r}(y)\}$ and $\{B(y), \mathbf{s}(y)\}$ be the integrated solutions of the problems (A.1)-(A.2) and (A.1)-(A.3), respectively; then there exist $D_{0} \overline{(A, y)} \overline{\in \mathbb{C}^{m_{1} \times m_{1}}}$ and $D_{1}(B, y) \in \mathbb{C}^{m_{2} \times m_{2}}$ such that

$$
\begin{array}{cc}
A^{\prime}=A M+D_{0} A, & A(0)=A_{0}, \\
\mathbf{r}^{\prime}=A \mathbf{f}+D_{0} \mathbf{r}, & \mathbf{r}(0)=\mathbf{r}_{0},
\end{array}
$$

and

$$
\begin{array}{cc}
B^{\prime}=B M+D_{1} B, & B(h)=B_{1}, \\
\mathbf{s}^{\prime}=B \mathbf{f}+D_{1} \mathbf{s}, & \mathbf{s}(h)=\mathbf{s}_{1} .
\end{array}
$$

Theorem A.2. If the two-point boundary value problem (A.1)-(A.3) has a unique solution, then the matrix

$$
\left[\begin{array}{l}
A(y) \\
B(y)
\end{array}\right] \in \mathbb{C}^{m \times m}
$$

is nonsingular.

Theorem A.3. The two-point boundary value problem (A.1)-(A.3) is equivalent to the linear system

$$
\left[\begin{array}{l}
A(y) \\
B(y)
\end{array}\right] \mathbf{u}(y)=\left[\begin{array}{l}
\mathbf{r}(y) \\
\mathbf{s}(y)
\end{array}\right]
$$




\section{APPENDIX B: TWO-POINT BOUNDARY} VALUE PROBLEM

In this section, we discuss the integration method for solving a two-point boundary value problem in detail. Consider the second-order boundary value problem

$$
\begin{gathered}
u^{\prime \prime}+\eta^{2} u=f, \quad 0<y<h, \\
u=0 \quad \text { at } y=0, \\
u^{\prime}-\mathrm{i} \eta u=g \quad \text { at } y=h .
\end{gathered}
$$

Let $v_{1}=u$ and $v_{2}=u^{\prime}$. The second-order boundary value problem (B.1)-(B.3) can be equivalently formulated into a first-order two-point boundary value problem:

$$
\begin{aligned}
& \mathbf{v}^{\prime}+M \mathbf{v}=\mathbf{f}, \\
& A_{0} \mathbf{v}(0)=0, \\
& B_{1} \mathbf{v}(h)=g,
\end{aligned}
$$

where

$$
\begin{aligned}
& \mathbf{v}=\left[\begin{array}{l}
v_{1} \\
v_{2}
\end{array}\right], \quad \mathbf{f}=\left[\begin{array}{l}
0 \\
f
\end{array}\right], \quad M=\left[\begin{array}{cc}
0 & -1 \\
\eta^{2} & 0
\end{array}\right], \\
& A_{0}=[10], \quad B_{1}=[-\mathrm{i} \eta 1] \text {. }
\end{aligned}
$$

Theorem B.1. The two-point boundary value problem (B.1)-(B.3) has a unique solution given by

$$
u(y)=K_{1}(y) g-\int_{0}^{h} K_{2}(y, z) f(z) \mathrm{d} z,
$$

where

$$
K_{1}(y)=\frac{e^{\mathrm{i} \eta} h}{2 \mathrm{i} \eta}\left(e^{\mathrm{i} \eta y}-e^{-\mathrm{i} \eta y}\right)
$$

and

$$
K_{2}(y, z)= \begin{cases}\frac{e^{\mathrm{i} \eta y}}{2 \mathrm{i} \eta}\left(e^{\mathrm{i} \eta z}-e^{-\mathrm{i} \eta z}\right), & z<y, \\ \frac{e^{\mathrm{i} \eta z}}{2 \mathrm{i} \eta}\left(e^{\mathrm{i} \eta y}-e^{-\mathrm{i} \eta y}\right), & z>y\end{cases}
$$

Proof. Since $M$ is a nonsingular matrix, there exists a nonsingular matrix $Q$ such that

$$
Q^{-1} M Q=N
$$

where

$$
\begin{aligned}
& N=\left[\begin{array}{cc}
-\mathrm{i} \eta & 0 \\
0 & \mathrm{i} \eta
\end{array}\right], \quad Q=\left[\begin{array}{cc}
1 & 1 \\
\mathrm{i} \eta & -\mathrm{i} \eta
\end{array}\right], \\
& Q^{-1}=\frac{1}{2 \mathrm{i} \eta}\left[\begin{array}{cc}
\mathrm{i} \eta & 1 \\
\mathrm{i} \eta & -1
\end{array}\right] .
\end{aligned}
$$

A simple calculation yields that the fundamental matrix of (A.4)-(A.5) is

$$
\Phi(y)=Q\left[\begin{array}{ll}
e^{\mathrm{i} \eta y} & \\
& e^{-\mathrm{i} \eta y}
\end{array}\right] Q^{-1},
$$

which gives

$$
\operatorname{det}\left[\begin{array}{c}
A_{0} \\
B_{1} \Phi(h)
\end{array}\right]=\left|\begin{array}{cc}
1 & 0 \\
-\mathrm{i} \eta e^{-\mathrm{i} \eta h} & e^{-\mathrm{i} \eta h}
\end{array}\right|=e^{-\mathrm{i} \eta h} \neq 0 .
$$

It follows from Theorem A.1 that the two-point boundary value problem (․․ $)-(\underline{B .6})$ and thus $(\underline{B .1})-(\underline{B .3})$ has a unique solution.

Let $\{A(y), r(y)\}$ and $\{B(y), s(y)\}$ be the integrated solutions of problems ( $\underline{\text { B.4 }}),(\underline{B} .5)$ and (B.4),$\underline{\text { B.6) }}$, respectively. Taking

$$
D_{0}=\mathrm{i} \eta, \quad D_{1}=-\mathrm{i} \eta,
$$

we obtain from Eqs. (A.7)-(A.10) that the integrated solutions satisfy

$$
A^{\prime}=A M+\mathrm{i} \eta A, \quad A(0)=A_{0},
$$

$$
r^{\prime}=A \mathbf{f}+\mathrm{i} \eta r, \quad r(0)=0,
$$

and

$$
B^{\prime}=B M-\mathrm{i} \eta B, \quad B(h)=B_{1},
$$

$$
s^{\prime}=B \mathbf{f}-\mathrm{i} \eta s, \quad s(h)=g .
$$

Upon solving the above initial value problem, we obtain the integrated solutions

$$
\begin{gathered}
A=\left[A_{1}, A_{2}\right]=\frac{1}{2 \mathrm{i} \eta}\left[\mathrm{i} \eta\left(1+e^{2 \mathrm{i} \eta y}\right), 1-e^{2 \mathrm{i} \eta y}\right], \\
B=\left[B_{1}, B_{2}\right]=[-\mathrm{i} \eta, 1],
\end{gathered}
$$

$$
r=\int_{0}^{y} e^{\mathrm{i} \eta(y-z)} A_{2}(z) f(z) \mathrm{d} z
$$

$$
s=e^{\mathrm{i} \eta(h-y)} g-\int_{y}^{h} e^{\mathrm{i} \eta(z-y)} f(z) \mathrm{d} z .
$$

It follows from Theorem A.3 that the two-point boundary value problem (B.4)-(B.6) is equivalent to the linear system

$$
\left[\begin{array}{ll}
A_{1} & A_{2} \\
B_{1} & B_{2}
\end{array}\right]\left[\begin{array}{c}
u \\
u^{\prime}
\end{array}\right]=\left[\begin{array}{l}
r \\
s
\end{array}\right]
$$


An application of Gram's rule yields

$$
u=\frac{r B_{2}-s A_{2}}{A_{1} B_{2}-B_{1} A_{2}} .
$$

A simple calculation yields

$$
A_{1} B_{2}-B_{1} A_{2}=1
$$

Substituting Eqs. (B.12)-(B.15) into Eq. (B.16), we deduce Eq. (B.7).

\section{ACKNOWLEDGMENTS}

The research of T. Cheng was supported in part by the NSFC (grant no. 11171126). The research of P. Li was supported in part by NSF grants DMS-1042958 and DMS-1151308.

\section{REFERENCES}

1. R. Petit, ed., Electromagnetic Theory of Gratings (SpringerVerlag, 1980).

2. G. Bao, D. Dobson, and J. A. Cox, "Mathematical studies in rigorous grating theory," J. Opt. Soc. Am. A 12, 1029-1042 (1995).

3. J. C. Nédélec and F. Starling, "Integral equation methods in a quasi-periodic diffraction problem for the time-harmonic Maxwell's equations,” SIAM J. Math. Anal. 22, 1679-1701 (1991).

4. Z. Chen and H. Wu, "An adaptive finite element method with perfectly matched absorbing layers for the wave scattering by periodic structures," SIAM J. Numer. Anal. 41, 799-826 (2003).

5. G. Bao, Z. Chen, and H. Wu, "Adaptive finite element method for diffraction gratings,” J. Opt. Soc. Am. A 22, 1106-1114 (2005).

6. G. Bao, L. Cowsar, and W. Masters, Mathematical Modeling in Optical Science, Vol. 22 of Frontiers in Applied Mathematics (SIAM, 2001).

7. A. Kirsch, "Uniqueness theorems in inverse scattering theory for periodic structures," Inverse Probl. 10, 145-152 (1994).

8. G. Bao, "A unique theorem for an inverse problem in periodic diffractive optics," Inverse Probl. 10, 335-340 (1994).

9. H. Ammari, "Uniqueness theorems for an inverse problem in a doubly periodic structure," Inverse Probl. 11, 823-833 (1995).

10. F. Hettlich and A. Kirsch, "Schiffer's theorem in inverse scattering theory for periodic structures," Inverse Probl. 13, 351-361 (1997).

11. G. Bao and A. Friedman, "Inverse problems for scattering by periodic structure," Arch. Ration. Mech. Anal. 132, 49-72 (1995).

12. G. Bao and Z. Zhou, "An inverse problem for scattering by a doubly periodic structure," Trans. Am. Math. Soc. 350, 4089-4103 (1998).

13. G. Bao, H. Zhang, and J. Zou, "Unique determination of periodic polyhedral structures by scattered electromagnetic fields," Trans. Am. Math. Soc. 363, 4527-4551 (2011).

14. G. Bruckner, J. Cheng, and M. Yamamoto, "An inverse problem in diffractive optics: conditional stability," Inverse Probl. 18, 415-433 (2002).
15. N. García and M. Nieto-Vesperinas, "Near-field optics inversescattering reconstruction of reflective surfaces," Opt. Lett. 24, 2090-2092 (1993).

16. K. Ito and F. Reitich, "A high-order perturbation approach to profile reconstruction: I. Perfectly conducting gratings," Inverse Probl. 15, 1067-1085 (1999).

17. T. Arens and A. Kirsch, "The factorization method in inverse scattering from periodic structures," Inverse Probl. 19, 1195-1211 (2003).

18. F. Hettlich, "Iterative regularization schemes in inverse scattering by periodic structures," Inverse Probl. 18, 701-714 (2002).

19. G. Bruckner and J. Elschner, "A two-step algorithm for the reconstruction of perfectly reflecting periodic profiles," Inverse Probl. 19, 315-329 (2003).

20. J. Elschner, G. Hsiao, and A. Rathsfeld, "Grating profile reconstruction based on finite elements and optimization techniques," SIAM J. Appl. Math. 64, 525-545 (2003).

21. G. Bao, P. Li, and H. Wu, "A computational inverse diffraction grating problem,” J. Opt. Soc. Am. A 29, 394-399 (2012).

22. G. Bao, P. Li, and J. Lv, "Numerical solution of an inverse diffraction grating problem from phaseless data," J. Opt. Soc. Am. A 30, 293-299 (2013).

23. D. Dobson, "Optimal design of periodic antireflective structures for the Helmholtz equation,” Euro. J. Appl. Math. 4, 321-340 (1993)

24. D. Dobson, "Optimal shape design of blazed diffraction grating," Appl. Math. Optim. 40, 61-78 (1999).

25. J. Elschner and G. Schmidt, "Diffraction in periodic structures and optimal design of binary gratings: I. Direct problems and gradient formulas," Math. Methods Appl. Sci. 21, 1297-1342 (1998).

26. J. Elschner and G. Schmidt, "Numerical solution of optimal design problems for binary gratings," J. Comput. Phys. 146, 603-626 (1998).

27. D. Courjon, Near-Field Microscopy and Near-Field Optics (Imperial College, 2003).

28. D. P. Nicholls and F. Reitich, "Shape deformations in rough surface scattering: cancellations, conditioning, and convergence," J. Opt. Soc. Am. A 21, 590-605 (2004).

29. D. P. Nicholls and F. Reitich, "Shape deformations in rough surface scattering: improved algorithms," J. Opt. Soc. Am. A 21, 606-621 (2004).

30. O. Bruno and F. Reitich, "Numerical solution of diffraction problems: a method of variation of boundaries," J. Opt. Soc. Am. A 10, 1168-1175 (1993).

31. A. Malcolm and D. P. Nicholls, "A field expansions method for scattering by periodic multilayered media," J. Acoust. Soc. Am. 129, 1783-1793 (2011).

32. P. Li and J. Shen, "Analysis of the scattering by an unbounded rough surface," Math. Methods Appl. Sci. 35, 2166-2184 (2012).

33. A. Malcolm and D. P. Nicholls, "A boundary perturbation method for recovering interface shapes in layered media," Inverse Probl. 27, 095009 (2011).

34. G. Bao and J. Lin, "Near-field imaging of the surface displacement on an infinite ground plane," Inverse Probl. Imag. 2, 377-396 (2013).

35. G. Bao and P. Li, "Near-field imaging of infinite rough surfaces," SIAM J. Appl. Math. (to be published).

36. G.-Q. Zhang, "Integrated solutions of ordinary differential equation system and two-point boundary value problems. I. Integrated solution method," J. Comp. Math. 3, 245-254 (1981). 